\title{
Detection of the unidentified infrared bands in a filament of the dwarf galaxy NGC1569
}

\author{
H. Matsumoto ${ }^{1}$, T. Onaka ${ }^{1}$, I. Sakon ${ }^{1}$, and H. Kaneda ${ }^{2}$ \\ ${ }^{1}$ Department of Astronomy, Graduate School of Science, The University of Tokyo, \\ Tokyo 113-0033, Japan \\ ${ }^{2}$ Institute of Space and Astronautical Science, Japan Aerospace Exploration Agency, \\ Kanagawa 229-8510, Japan
}

\begin{abstract}
We made near- to mid-infrared imaging and spectroscopic observations of the dwarf galaxy NGC 1569 with the Infrared Camera (IRC) on board AKARI. The unidentified infrared (UIR) band features at $6.2,7.7$, and $11.2 \mu \mathrm{m}$, which are generally attributed to polycyclic aromatic hydrocarbons (PAHs), are clearly detected in a structure associated with an $\mathrm{H} \alpha$ filament. The filament is filled with X-ray emission and is thought to be formed by outflow from the galaxy. Since PAHs are destroyed rapidly in hot plasma, it is most likely that PAHs in the filament are produced from fragmentation of large carbonaceous grains in the shock. We also detect excess emission in $2-5 \mu \mathrm{m}$ in the filament, which may come from very small grains.
\end{abstract}

Keywords. Infrared: galaxies, dust, extinction, galaxies: individual (NGC1569)

\section{Introduction}

The unidentified infrared (UIR) bands, whose major features appear at 3.3, 6.2, 7.7, 8.6, and $11.2 \mu \mathrm{m}$, are seen in the diffuse Galactic emission as well as in external galaxies. Polycyclic aromatic hydrocarbons (PAHs) or PAH-like aromatic groups of carbonaceous materials are thought to be the carriers of the UIR bands. There remains an interesting question where and how the band carriers are produced in galaxies, in particular in dwarf galaxies, which may represent the characteristics of the early universe because of their low-metallicity. We report here the results of near- to mid-infrared imaging and spectroscopic observations of the starburst dwarf galaxy NGC 1569 with the Infrared Camera (IRC) on board AKARI (Murakami et al. 2007). NGC 1569 is a well-studied nearby galaxy with the metallicity of about a quarter of solar, which harbors several super star clusters (SSCs) in it (e.g., Tokura et al. 2006). A number of filamentary structures are detected in $\mathrm{H} \alpha$ (Hunter et al. 1993), which delineate the X-ray emission. It is suggested that the interaction between the galactic wind and the surrounding gas results in superbubbles and $\mathrm{H} \alpha$ emitting shocked gas (Martin 1998, Martin et al. 2002). There is a HI cloud about $5 \mathrm{kpc}$ from the galaxy, which may fuel HI gas into NGC 1569 (Stil \& Israel 1998, Mühle et al. 2005). We report here the detection of the UIR bands in one of the $\mathrm{H} \alpha$ filaments and discuss the possible supply source of the band carriers.

\section{Observations}

NGC 1569 was observed in the imaging (at 3, 4, 7, 11, 15, and $24 \mu \mathrm{m}$ ) and spectroscopic $(2-13 \mu \mathrm{m})$ modes of the IRC (Onaka et al. 2007, Ohyama et al. 2007) on 2006 September 9 and on 2007 March 8, respectively. A western filament appears prominently in the $7 \mu \mathrm{m}$ image (Figure 1a), which correlates well with the $\mathrm{H} \alpha$ emission (Hunter et al. 1993), while structures corresponding to the filament are not clearly seen in the 15 and $24 \mu \mathrm{m}$ images. Since the $7 \mu \mathrm{m}$ band probes the UIR 6.2 and $7.7 \mu \mathrm{m}$ bands efficiently (Sakon 
et al. 2007), this result suggests the presence of the UIR bands in the filament and subsequent spectroscopic observations of the filament were carried out. The spectrum of the western filament is shown in Figure $1 \mathrm{~b}$ after subtracting the sky background. It confirms the presence of the UIR bands at $6.2,7.7$, and $11.2 \mu \mathrm{m}$ in the filament. There may be weak emission at 3.3 and $8.6 \mu \mathrm{m}$, but further observations are needed to confirm their presence.
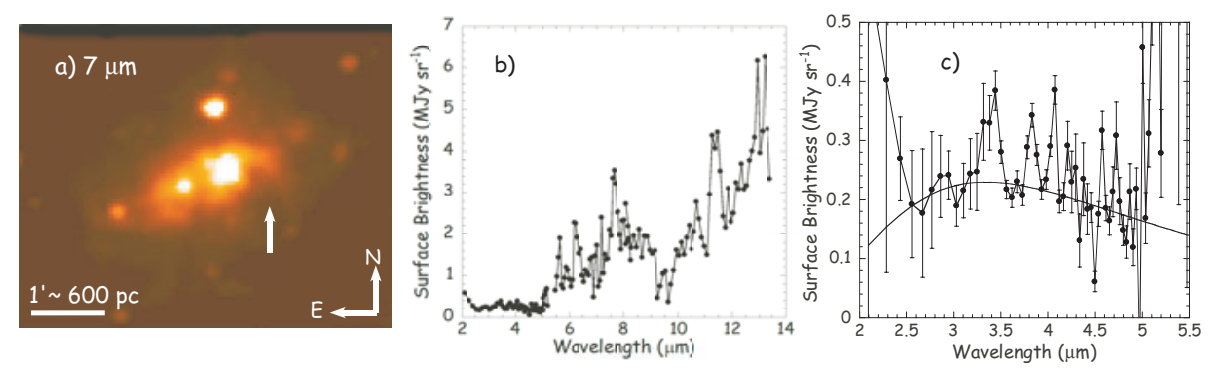

Figure 1. (a) $A K A R I /$ IRC $7 \mu \mathrm{m}$ image of NGC 1569. The white arrow indicates the western filament. (b) IRC spectrum of the filament. (c) An enlargement of the near-infrared spectrum of the filament. The solid line indicates a fit with a blackbody $(868 \mathrm{~K})$ with a $\lambda^{-2}$ emissivity.

\section{Discussion}

The UIR bands have been detected in halos of galaxies (Irwin \& Madden 2006, Irwin et al. 2007) and in filaments produced by outflows (Tacconi-Garman et al. 2005) based on imaging observations. The present observations detect the UIR $6.2,7.7$, and $11.2 \mu \mathrm{m}$ bands in a filamentary structure of a galaxy by spectroscopy for the first time. Since PAHs are destroyed rapidly in hot plasmas, it is most likely that PAHs in the filament are produced by fragmentation of large carbonaceous grains in the shock. Figure 1c also suggests the presence of excess continuum emission at $2-5 \mu \mathrm{m}$ in the filament. Similar excess emission, which is possibly due to very small grains, has been observed in external galaxies (Lu et al. 2003) as well as in the diffuse Galactic emission (Flagey et al. 2007).

The work is based on observations with $A K A R I$, a JAXA project with the participation of ESA. It is supported by Grants-in-Aid for Scientific Research from MEXT and JSPS.

\section{References}

Flagey, N. et al. 2006, A\&A, 453, 969

Hunter, D. A., Hawley, W. N., \& Gallagher, J. S. 1993, AJ, 106, 1797

Irwin, J. A., Kennedy, H., Parkin, T., \& Madden, S. 2007, A\&A, 474, 461

Irwin, J. A. \& Madden, S. 2006, A\&A, 445, 123

Lu, N. et al. 2003, ApJ, 588, 199

Martin, C. L. 1998, ApJ, 491, 561

Martin, C. L., Kobulnicky, H. A., \& Heckman, T. M. 2002, ApJ, 574, 663

Murakami, H. et al. 2007, PASJ, 59, S369

Mühle, S., Klein, U., Wilcots, E. M., \& Hüttenmeister, S. 2005, AJ, 130, 524

Ohyama, Y. et al. 2007, PASJ, 59, S411

Onaka, T. et al. 2007, PASJ, 59, S401

Sakon, I. et al. 2007, PASJ, 59, S483

Stil, J. M. \& Israel, F. P. 1998, A\& $A, 337,64$

Tacconi-Garman, L. E. et al. 2005, A\&A, 432, 91

Tokura, D. et al. 2006, ApJ, 648, 355 